\title{
MapReduce Implementation Based on vStarCloud
}

\author{
Zhu Lei, Chen Zuo-Ning, and Yin Hong-Wu
}

\begin{abstract}
As a lot of cloud computing platforms are being developed, the number of cloud computing platform is increasing rapidly, with various forms. Therefore, collaborative integrated development is necessary for the entire cloud computing ecosystem to establish and enrich. Therefore, the vStarCloud is proposed, which is a cloud computing operating system architecture based on the idea of distributing and layering. This paper introduces a method of the deployment and implementation of MapReduce application based on vStarCloud, and the feasibility of vStarCloud will be verified by experiment. Also, the performance of MapReduce with Hadoop will be compared.
\end{abstract}

Index Terms-vStarCloud, MapReduce, cloud operating system.

\section{INTRODUCTION}

With the rapid development of the Internet technology, the new network application model, with cloud computing as the representative, has brought a series of challenges to computer technology, especially the software technology. There are a lot of architectures and methods of cloud computing, but none of them bring cloud facilities into a unified, scalable, self-management, and reliable computing architecture [1]-[3].

In order to solve the problem of the integration development of various cloud computing systems, the vStarCloud cloud operating system architecture is proposed. It can build a cloud computing service platform with a high-level of resource management and sharing, supporting diversity and flexibility of service and a variety of new network application modes. This paper researches the feasibility and availability of the vStarCloud, by developing MapReduce application on it, and comparing the performance of MapReduce with Hadoop.

\section{OVERVIEW OF CLOUd OPERATING SYSTEM}

The cloud operating system is a new word that is proposed recent years in the cloud computing field. It is mainly used to manage the various resources in cloud computing environment.

Manuscript received December 12, 2012; revised April 10, 2013. This work is supported by the National Key Science and Technology Research Program of China under Grant No.2011X01039-001-002.

Zhu Lei is with the Jiangnan Institute of Computing Technology, Postgraduate, Wuxi, China (e-mail: 3613807@ 163.com).

Chen Zuo-Ning is with the Jiangnan Institute of Computing Technology, the academician of Chinese Academy of Engineering (e-mail:chenzuoning@163.net)

Yin Hong-Wu is with the Jiangnan Institute of Computing Technology, Senior Engineer, Wuxi, China (e-mail: 13812092679@139.com).

\section{A. Concept}

As known to all, the computer operating system is mainly used to manage a variety of hardware resources, such as CPU, memory, etc. It transparencies the details operation of hardware to users, and realizes effective sharing of resources. Building the cloud operating system has the same purpose with building the computer operating system, but the difference is that, the cloud operating system is to manage cloud facilities, it transparencies the concrete operation of cloud facilities to users, and implements sharing of these limited resources.

Fabio Pianese etc. [4] define cloud computing operating system as follows:

The cloud operating system aims to provide a familiar interface for developing and deploying massively scalable distributed applications on behalf of a large number of users, exploiting the seemingly infinite CPU, storage, and bandwidth provided by the cloud infrastructure.

\section{B. Characteristics}

Cloud operating system is the extension of the meaning of the operating system in the cloud computing environment. Comparing with the computer operating system, difference in scale and realization are ignored, the characteristics of the cloud operating system are summarized as follows [4]:

Computer operating system consists of a series of programs, such as scheduler, file system, interrupt handlers, etc. These programs are used to manage software interfaces of CPUs, memory, disks and other external hardware devices; Cloud computing operating system provides an additional set of functionalities that enables administrators to get access to resources in the cloud, including allocating and deallocating virtual machines, dispatching and migrating processes, creating inter-process communication, etc.

Computer operating system provides the standard library of system calls, which can be used by programs to access the underlying hardware; cloud operating system provides a set of interfaces based on network, which can be used by applications to query management system and control cloud resources.

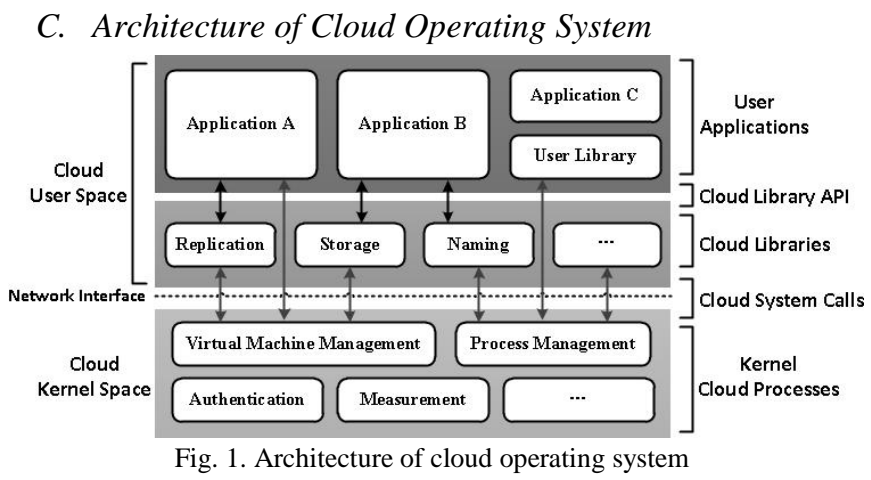


Computer operating system provides a standard distribution of libraries and software packages; Cloud operating system provides software support for the autonomous scaling and deployment of distributed applications.

Fig. 1 shows the architecture of the cloud operating system, Fabio Pianese, etc. [1] define two basic logical definitions of this architecture.

Cloud Objects: A set of local operating system processes running on a single node, which are wrapped together and assigned locally a random identifier of suitable length to minimize the risk of system-wide ID collisions.

Cloud Process: A collection of Cloud objects that implement the same (usually distributed) application.

According to the differences of how applications are running on cloud process, cloud operating system architecture is divided into the following three parts:

Cloud Kernel Space: A small number of cloud processes that regulate physical allocations, access controls, accounting, and measurements of resources.

Cloud User Space: These cloud processes that do not belong to the cloud kernel space process. The cloud user space consists of the user application, API libraries and standard library.

Network Interface: It is used for interactive between cloud kernel space and the cloud user space.

In the cloud operating system, cloud kernel space needs to realize the implementation of resources scheduling and abstract, the management of distributed processes and applications, as well as access control user identity authentication; cloud user space need to provide a standard set of interfaces for developers to meet the common needs of distributed large-scale applications.

\section{VSTARCLOUD}

vStarCloud is a cloud operating system developed by Jiangnan Institute of Computing Technology etc. It aims at solving the problems of scalability, flexibility, credibility etc. in cloud computing.[5]

\section{A. Designing Goals of vStarCloud}

By analyzing the demands of the users of cloud operating system, we summarized some basic demands as follows:

- Cloud operating system must be able to manage the resources in the cloud environment.

- Cloud operating system must support large-scale system management and running maintenance;

- Cloud operating system must have the feature of high availability, like continuing to provide service in the case of node failure and network failure;

- Cloud operating system must have the versatility, supporting as many types of applications as possible, and providing good compatibility;

- Cloud operating system should be distributed, scalable, and cost-effective;

- Cloud operating system must have security, eliminating the security risks brought about by the new mode of computing and storage as much as possible.

Therefore, vStarCloud aims at solving the problem of integrated development of various cloud computing platforms, and meeting users' demands as using a cloud computing service platform with a high-level of resource management and sharing, supporting diversity and flexibility. By managing a variety of hardware and software resources, there are abstracted to the cloud task and the cloud storage, it is able to make full use of distributed technology to manage effectively, provide standard system call interface and library to the upper user, and also support a variety of new network application modes.

\section{B. vStarCloud Cloud Operating System Architecture}

By analyzing of the logical architecture of the cloud operating system, considering the design requirements, design of vStarCloud cloud operating system architecture is shown in Fig. 2.

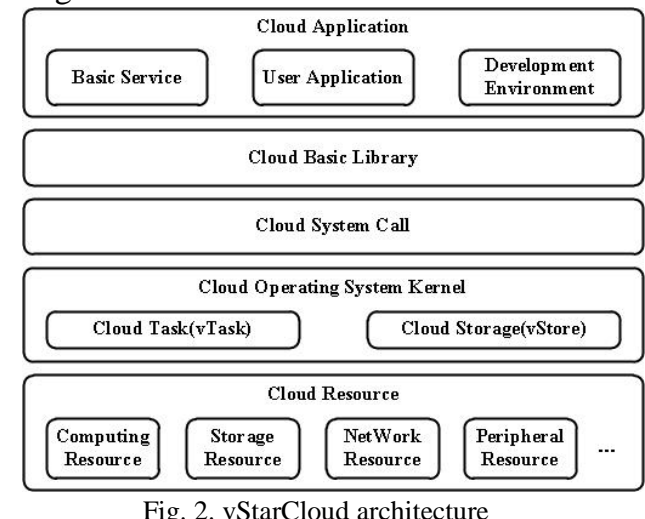

Objects involved in each levels of the architecture of vStarCloud are as follows:

Cloud Resource: All available physical resources in the cloud computing environment are called cloud resources, including computing resources, storage resources, network resources, and other peripheral resources. Computing resources typically contains processor resources, memory resources; storage resources include node local storage, global storage resources, etc. network resources include node network resources, switches, routers, and other network resources; peripheral resources mean some special equipment resources in cloud environment, such as printers, special equipment, etc.

Cloud Task (vTask): An application task using cloud resources to complete a common goal is called a cloud task. Cloud task is the scheduling physical units within the cloud operating system, generally having network communication capability. When the cloud platform provides services as IaaS form, a typical cloud task is the machine virtual machine (Machine virtual machine, Machine VM), when the cloud platform provides services as the form of PaaS, a typical cloud task is a user program developed using some certain languages.

Cloud Storage (vStore): The entity providing cloud persistent storage capacity is called vStore. Usually it is a logical storage form that specifically built upon the storing physical resources for cloud tasks in cloud resources. Meanwhile, it can have different functional cloud storage forms for the different needs of the cloud task.

Cloud Operating System Kernel: it provides cloud resources management (access control, monitoring, applying, 
release, etc.), cloud task lifecycle management (create, deployment, scheduling, destruction, etc.), as well as cloud storage support.

Cloud System Call: it is the basic interfaces provided by cloud kernel used by the user layer of cloud operating system. It usually completes the management functions such as applying to kernel for releasing resources, and interacting with kernel, etc. The cloud system call is not been packaged. It is the most original, the most direct interaction with the cloud kernel interface form.

Cloud Basic Library: it is a special interface library for the convenience of users so that they can develop and run the programs. It is usually packaging cloud system calls, and providing to users with various forms.

Cloud Basic Services: these cloud services are present in the cloud operating system environment, to make users to use directly without developing.

Cloud Application: cloud applications are developed by users with cloud library or cloud system call, or repackage from a variety of cloud basic services.

\section{Application Implementation Based on vStarCloud}

The architecture of vStarCloud application is shown in Fig. 3.

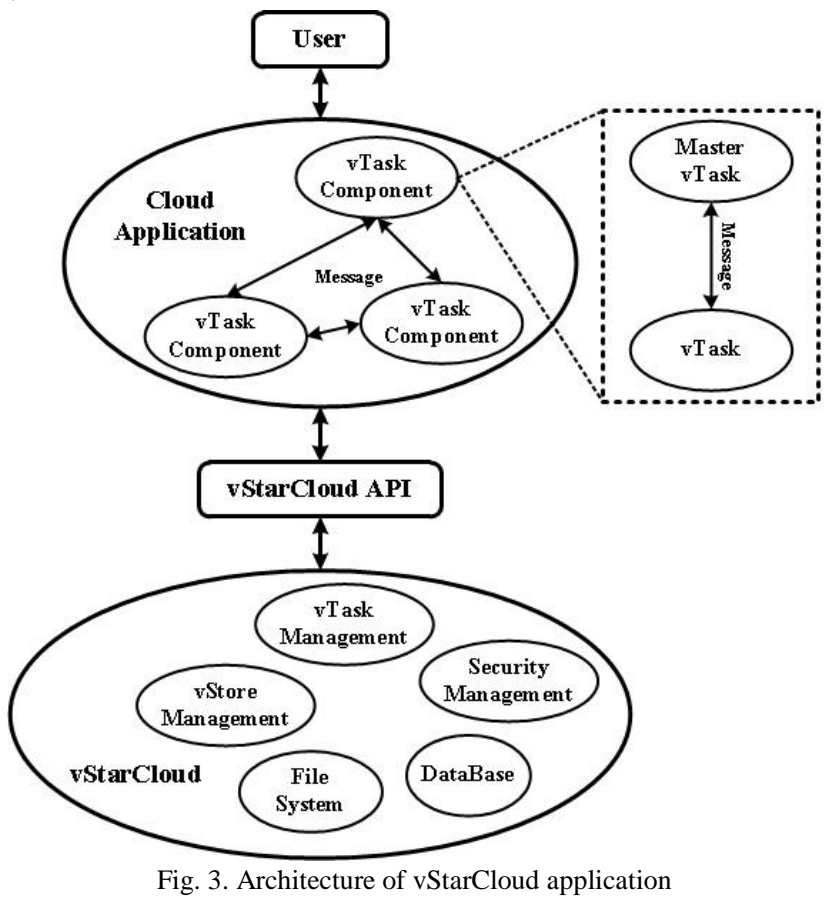

A vStarCloud application is consisted of many cloud tasks. These cloud tasks will be interacting with infrastructure, such as file system, database, etc. by vStarCloud internal interface.

Messages are very important to cloud application implementation. Operations need to be delivered by messages, such as deploying, monitoring and managing cloud task, passing the compute result, etc. vStarCloud has mature message queue mechanism. It can not only implement point-to-point communication, but also broadcast messages. Thus, it can greatly reduce systemic coupling factor, and improve the flexibility of vStarCloud.

It is easily to be seen from Fig. 3 that it's implementing a specific application, the most critical part is the using of cloud task component. The cloud task is divided into two parts:

\section{Master vTask and vTask.}

The Master vTask plays the role of deploying, monitoring of vTask. The Master vTask is the transition between the top and the bottom, playing the role of a "nexus". It receives a command from the upper, deploys vTask to complete the users' wish of completing an application. At the same time, after the completion of the vTask execution, feeding back timely the results to users.

vTask is a specific application to meet the user requirements to implement the program. Any application is completed through the execution of series of vTasks at the bottom. It is the cloud computing entity scheduling unit in vStarCloud. Referring to the architecture of cloud operating system introduced before, vTask is actually the cloud objects defined in the architecture. It is composed by a set of progress where it is situated in the calculating node. Through the completion of the application required by the user by deploying vTasks, and passing results in a timely manner to the Master vTask, and then informing the users, the implementation of an application is completed.

\section{Analysis of Cloud Task}

Cloud tasks are the key of the vStarCloud cloud operating system, any application is completed by deploying cloud task. The cloud task is defined as follows:

- Cloud tasks are the scheduling entities that can communicate with each other. Though multiple cloud tasks working, a cloud application can be completed in a distributed environment.

- Actually, cloud task is a small "container", it can store cloud resources independently. According to the difference of user needs, cloud task can implement different content, such as virtual machine, programing environment, etc. The multiple clouds tasks combine together to complete the application required by the user.

- A cloud task actually corresponds to a group of associated processes in a physical node, these processes operate in conjunction to complete the cloud task.

The cloud task has some characteristics as follows:

Dynamic: Cloud task has a certain life cycle, not permanent existing. It runs with creation, and destroys with execution finished.

Concurrency: Multiple cloud tasks can co-exist, and run simultaneously during a period of time. They may either be in order to accomplish the same service, or to complete different services.

Interoperability: Message mechanism exists not only among cloud tasks, but also between the master vTask and the vTask. Cloud task interacts with the upper through messages.

Independence: Cloud task is a basic unit that operates separately, allocates resources separately, and accepts scheduling independently. Cloud tasks work independent, other cloud tasks can't affect it.

\section{IMPLEMENTATION OF MAPREDUCE BASED ON VSTARCLOUD}

MapReduce is being the most classical application provided by PaaS service. The key point is that, MapReduce realized parallel computing of large-scale data set in 
distributed platform, providing a parallel programming model of mass data. By using this model, it is easy to write distributed parallel programs. Users can develop distributed parallel program conveniently, even without the experience of parallel development, and complete the calculation of mass data.[6]

\section{A. Description of Implementation}

The implementation architecture of MapReduce is mainly composed of the following components: Master vTask, Map Worker and Reduce Worker. Map Worker and Reduce Worker are constituted by a series of cloud tasks. Also, they need to collaborate with the file system of vStarCloud work to complete MapReduce. In order to compare the performance of MapReduce with that of Hadoop, analysis of the implementation architecture of WordCount and the classic application of Hadoop MapReduce are the first things to do.

Fig. 4 shows the Hadoop MapReduce computing processes.

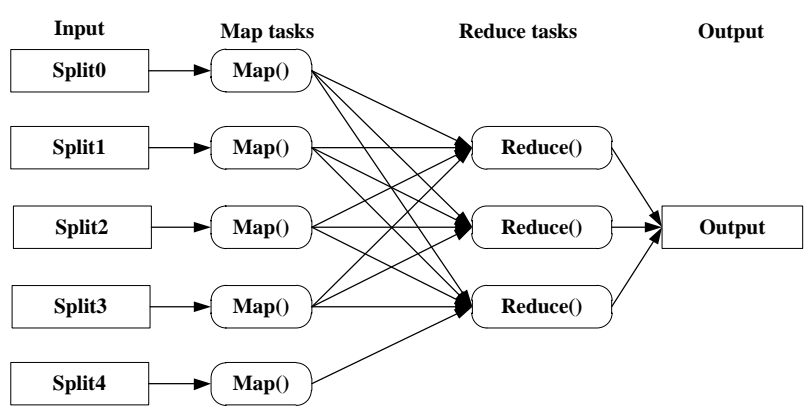

Fig. 4. Hadoop MapReduce

For the WordCount application, MapReduce mainly processes in the following three steps:

a) According to information contained in file, the file is divided into the $<$ key, value $>$ form;

b) Each < key, value $>$ pair which is split is processed by the user-defined Map function, and then new $<$ key, value $>$ pairs are generated;

c) < key, value $>$ pair exported by Map progress is sent to the user-defined Reduce function to process, and the new $<$ key, value $>$ pair exported is the final result..

Fig. 5 shows the vStarCloud MapReduce architecture.

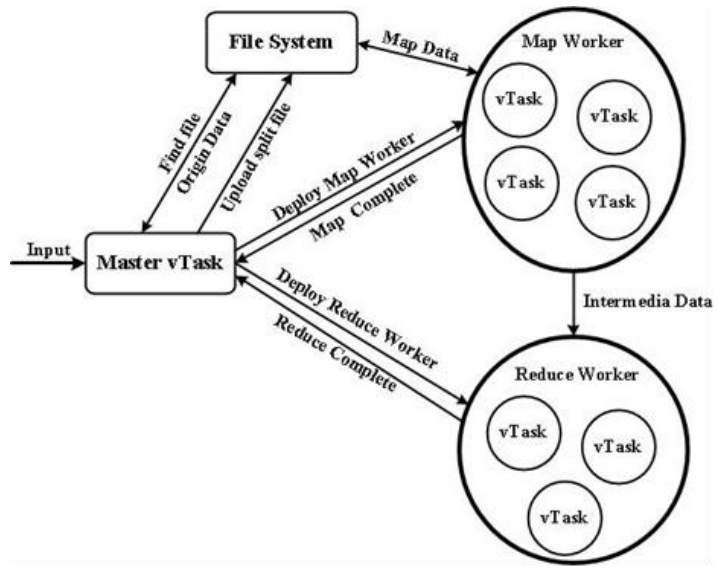

Fig. 5. vStarCloud MapReduce

As it can be seen from Fig. 5, the key of MapReduce implementation is the use of cloud task in vStarCloud. In MapReduce procedure, users operate the master vTask, and the master vTask deploys the concrete cloud tasks by message mechanism. Each cloud task corresponds to a map task or a reduce task. When completed, each cloud task returned the computing result to the Master vTask by message, then, the entire MapReduce is finished. For WordCount application, vStarCloud MapReduce goes with the following three steps:

a) According to the file path provided by user, the Master vTask first downloads this file in file system file, and then, splits file according to user requirement of the file size, then passes these split file to file system. Then, it creates cloud tasks which are map tasks and reduce tasks, and pass the $<$ key, value $>$ pair to map tasks, with split file name as its key, and the file path as its value.

b) After creating map tasks, the file in the file system needs to be find according to the incoming <key,value> pair in map task. Then, after handling this file, the new <key,value $>$ pair is passed to reduce task by message. Meanwhile, cloud task sends message to the master vTask when the cloud task finishes. The master vTask will send the stop command to the map task.

c) In the reduce task, After the incoming <key,value> pair is received from map task, it is handled by user-defined reduce function, and then, the result is sent to the master vTask. After receiving this message, the master vTask will combine the results, export the final result, and send the stop command to the reduce task.

\section{B. Experimental Evaluation}

The experiment compares the performance of the WordCount application between Hadoop 1.0.0 and vStarCloud v1.0. They are deployed in the same experimental environment. Two different data files are used with the size $1 \mathrm{G}$ and $5 \mathrm{G}$, which are split by $64 \mathrm{M} /$ block.

It needs to be noted that, the interface of cloud task is realized by python language, so that map and reduce functions use Python language to write. In order to make an available comparison, Hadoop does not use the Java language or calls the Hadoop built-in class to complete MapReduce. Instead, it uses Python language to write map and reduce function, and it completes MapReduce in the form of Hadoop streaming.

The results of WordCount application are shown in Table I.

\begin{tabular}{lll}
\multicolumn{3}{l}{ TABLE I: EXPERIMENTAL RESULTS OF RUN TIME } \\
\hline \hline Data file & vStarCloud & Hadoop \\
\hline $1 \mathrm{G}$ & $421 \mathrm{~s}$ & $438 \mathrm{~s}$ \\
$5 \mathrm{G}$ & $1817 \mathrm{~s}$ & $2062 \mathrm{~s}$ \\
\hline \hline
\end{tabular}

It is easy to see from Table I that the WordCount performance of vStarCloud is slightly better than that of Hadoop, and with the file size increasing, the performance advantage is more obvious.

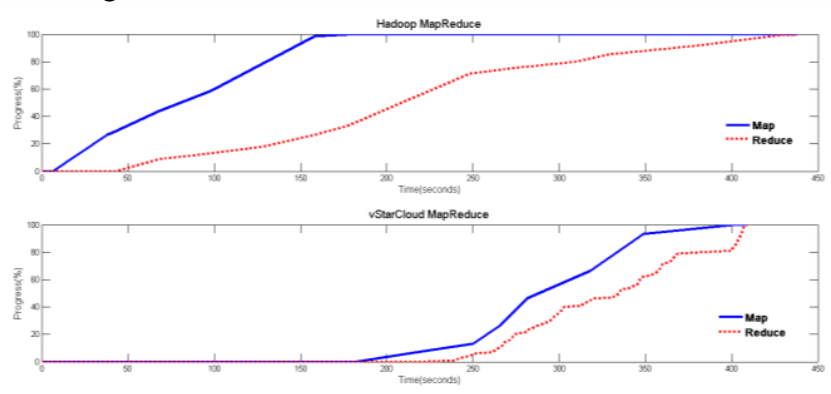

Fig. 6. Computation progress for Hadoop and vStarCloud. 1GB data 


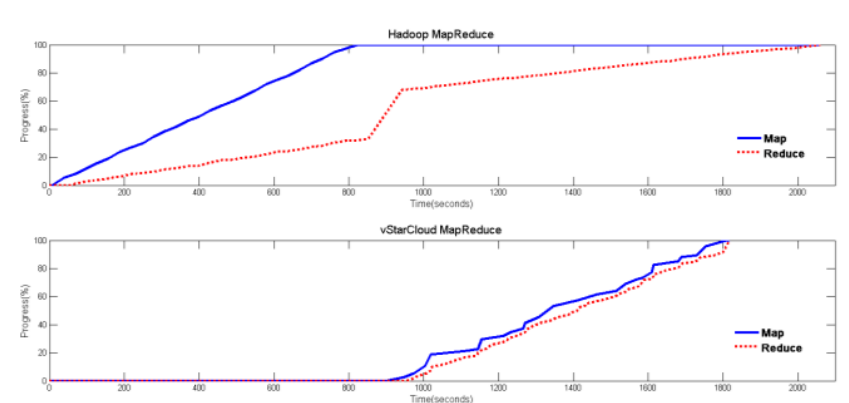

Fig. 7. Computation progress for Hadoo and vStarCloud. 5GB data

As shown in these figures, the operation conditions of the two cloud platforms have some similarities, as well as have some obvious differences. Now, we analyze the reasons as follows.

To complete MapReduce, three stages are needed, which are preparatory stage, MapReduce stage, and output stage. Therefore, the total runtime can be presented by the formula:

$$
T_{\text {total }}=T_{\text {ready }}+T_{\text {mapreduce }}+T_{\text {output }}
$$

In the preparatory stage, the time needed for Hadoop is very short while for vStarCloud very long. The reason is that Hadoop owns its shared file system HDFS. When MapReduce runs in Hadoop, it can read files from HDFS directly, and do some work like splitting files. But vStarCloud is depended on existing mature file system, as it has not yet developed its own shared file system. Therefore, when MapReduce is running in its early stage, vStarCloud needs to complete many work, such as downloading files, splitting files, and uploading files, etc. So, vStarCloud needs more time in preparatory stage.

In the MapReduce stage, as shown in the figures, map tasks always begin earlier than reduce tasks for both of the cloud platforms. The difference is that, for Hadoop, map tasks complete very quickly, while reduce tasks require a very long time. But for vStarCloud, map and reduce tasks complete almost at the same time. The two cloud platforms use the same algorithm, that is to say, the number of messages passed between the map and reduce tasks is the same. Thus, it illustrates that the message mechanism of vStarCloud is better than Hadoop obviously.

In the output stage, both of the two cloud platforms take a relatively short time. Therefore, there is not obvious gap between them.

From the specific data, take the $5 \mathrm{G}$ data file as an example, the two cloud platforms complete MapReduce by 75 map tasks and one reduce tasks. The total runtime of Hadoop is:

$$
T_{\text {total }}=T_{\text {ready }}+T_{\text {mapreduce }}+T_{\text {output }}=7 \mathrm{~s}+2050 \mathrm{~s}+5 \mathrm{~s}=2060 \mathrm{~s}
$$

In MapReduce stage, the total runtime of the map tasks is $814 \mathrm{~s}$, and time for reduce task is $2002 \mathrm{~s}$, the average runtime of each map task is $41 \mathrm{~s}$.

The total runtime of vStarCloud is:

$$
T_{\text {total }}=T_{\text {ready }}+T_{\text {mapreduce }}+T_{\text {output }}=898 s+915 s+4 s=1817 \mathrm{~s}
$$

In MapReduce stage, the total runtime of the map tasks is $868 \mathrm{~s}$, and time for reduce tasks is $911 \mathrm{~s}$, the average runtime of each map task is 49 s.
It can be seen from the previous data, during the MapReduce stage, time that Hadoop map tasks used is significantly less than that of reduce tasks. The reason is that, data that map tasks demand can be read from HDFS directly, but data that reduce tasks demand is exported from map tasks, passed by messages, and then handled by reduce tasks after received, thus, the system bottleneck is produced The lager the data file size is, the more obvious the bottleneck is. However, time that vStarCloud map tasks and reduce tasks used are almost the same, that is to say, for vStarCloud,once the map task exports a value, the reduce task receives and deals with it. Meanwhile, for single map task runtime, Hadoop is better than vStarCloud, the reason is also that vStarCloud doesn't have its own file system. When each map task is executed in vStarCloud, it first needs to download data file that is split from file system. If this time is not counted, both of the runtime of two cloud platforms map tasks are almost equal.

This experiment proves that, MapReduce based on vStarCloud cloud operating system can achieve good performance.

As it can be proved by this experiment, MapReduce developed based on vStarCloud can obtain good performance. With further research and development, after having its own file system, the performance of vStarCloud should be on a higher level.

\section{SUMMARY}

Cloud computing model has gained a huge success in business, but the specific form and effective way to accomplish cloud computing has not come to a conclusion. Cloud operating system plays the fundamental supporting role in the cloud computing system software stack. This paper analyzes the cloud operating system concepts and characteristics, as well as the independently developed vStarCloud cloud operating system, and implements MapReduce applications based on vStarCloud.

We believe that, with the concept of cloud operating system becoming gradually clear and the cloud architecture based on vStarCloud being proposed, in the near future, cloud computing ecosphere is able to build based on, the development of cloud computing will rise to a new height.

\section{REFERENCES}

[1] B. Rochwerger, A. Galis, D. Breitgand et al., "Design for future internet service infrastructures," Future Internet Assembly 2009, Prague, Czech Republic, in Towards the Future Internet - A European Research Perspective, pp. 227-237, IOS Press, May 2009.

[2] G. V. Laszewski, J. Diaz, F. Wang, and G. C. Fox, "Comparison of Multiple Cloud Frameworks," in Proc. IEEE Fifth International Conference on Cloud Computing, 2011.

[3] A. Baumann, S. Peter, A. Schüpbach, A. Singhania, T. Roscoe, P. Barham, and R. Isaacs, "Your computer is already a distributed system., Why isn't your OS?," in Proc. the 12th Workshop on Hot Topics in Operating Systems, May 2009

[4] F. Pianese, P. Bosch et al., "Toward a Cloud Operating System," IEEE, 2010 .

[5] Z. Chen, H. Yin et al., "vStarCloud: A New Network Computing Operating System for Multicore and Clouds," in Proc. Fourth International Symposium on Parallel Architectures, Algorithms and Programming, 2011. 
[6] H. Liu and D. Orban, "Cloud MapReduce: a MapReduce Implementation on top of a Cloud Operating System," in Proc. 11th IEEE/ACM International Symposium on Cluster, Cloud and Grid Computing, 2011.

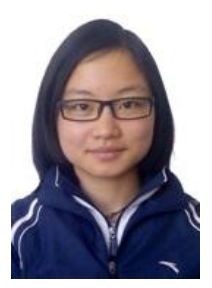

Zhu Lei was born in Chongqing, China, in 1989. She is the postgraduate of Jiangnan institute of computing technology in Wuxi. Her major field of study is computer operating system and cloud computing.

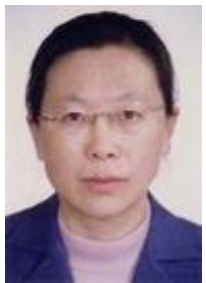

Chen Zuoning was born in Beijing in 1957. She is the academician of Chinese Academy of Engineering. Her major field of study is computer architecture and system software. She is the chief engineer of Jiangnan institute of computing technology in Wuxi. She is part-time professor and doctoral tutor of many well-known colleges. In recent years, she has undertaken and completed 3 national projects, 1 provincial projects, published 5 papers in important academic journals, published 2 monographs. She is CCF executive vice chairman and expert in national 863 program (information technology).

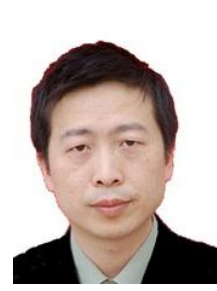

Yin Hongwu was born in Hunan, China, in 1973. He is the senior engineer of Jannan institute of computing technology in Wuxi. His major field of study is computer operating system and system software. 